\title{
Ten-year experience with transcatheter aortic valve implantation in bicuspid aortic valve: lessons learned and future perspectives
}

\author{
Michał Walczewski, Aleksandra Gasecka, Zenon Huczek, Bartosz Rymuza, Janusz Kochman \\ $1^{\text {st }}$ Chair and Department of Cardiology, Medical University of Warsaw, Warsaw, Poland
}

Adv Interv Cardiol 2021; 17, 3 (65): 251-258

DOI: https://doi.org/10.5114/aic.2021.109226

\begin{abstract}
A bstract
Bicuspid aortic valve (BAV) stenosis has traditionally been perceived as a contraindication to transcatheter aortic valve implantation (TAVI) due to its specific anatomical characteristics including extensive calcifications, high leaflet coaptation and frequently encountered aortic root dilation, which may result in worse procedural outcomes and higher risk of complications. Hence, BAV patients were not included in previous clinical trials. In the recent years, improved pre-procedural imaging and technological advances have gradually enabled expansion of TAVI to patients with complex anatomy, including those with BAV. Moreover, indications for TAVI are expanding to a younger group of patients with fewer comorbidities, and BAV is more prevalent in this population. Contemporary multicenter registry-based studies indicate that patients undergoing TAVI for BAV have similar outcomes as those with tricuspid aortic valve stenosis. In this article, we provide a thorough overview of the available clinical data regarding the outcomes of TAVI in BAV, from the perspective of an experienced TAVI center with over 150 TAVIs in this group of patients, performed in our institution since the year 2009. We present anatomical and clinical classifications of BAV, differences in outcomes in patients with bicuspid and tricuspid valves, as well as important topics regarding choice of an adequate valve and valve size.
\end{abstract}

Key words: aortic stenosis, transcatheter aortic valve implantation, bicuspid aortic valve.

\section{Introduction}

Bicuspid aortic valve (BAV) is the most common congenital anomaly in adults, with a frequency of $1-2 \%$ of the total population [1]. It is associated with accelerated degeneration of the valve apparatus that usually starts at younger age and tends to be more severe than in tricuspid aortic valve (TAV). Rapid calcium deposition, premature fibrosis and stiffening result in the development of aortic stenosis (AS) and aortic regurgitation (AR) even 10 years earlier than in TAV [2]. Thus, patients with BAV may require valve replacement at an earlier age. Along with the probable future expansion of transcatheter aortic valve implantation (TAVI) to a younger group of patients with AS, BAV will become more prevalent amongst patients undergoing TAVI. Previously, BAV was considered as a contraindication to TAVI due to anatomical differences, challenging even for experienced operators. Apart from the problems with the valve apparatus itself, aneurysms, dissections, coarctations and larger diameter of the ascending aorta are common findings in patients with BAV. Almost $40 \%$ of these patients have dilation of the aortic root [3], and although the risk of aneurysm and aortic dissection is much higher than in patients without BAV anatomy [4], life expectancy does not differ from that of the general population [5, 6]. Nevertheless, 2 large studies proved that nowadays life expectancy of BAV patients does not differ from the general population $[5,6]$. Similarly, recent multicenter registry-based studies indicate that patients with BAV stenosis have similar TAVI outcomes as patients with TAV stenosis [7-10]. With over 150 TAVI procedures in BAV since 2009 and more than 10 years of follow-up, our institution has developed substantial expertise with this patient population. Here, we provide a thorough overview of the available clinical data regarding the outcomes of $\mathrm{TAVI}$ in $\mathrm{BAV}$, discuss lessons we have learned and provide some future perspectives.

Corresponding author:

Prof. Janusz Kochman MD, PhD, $1^{\text {st }}$ Chair and Department of Cardiology, Medical University of Warsaw, Warsaw, Poland,

phone: +48 2259919 51, e-mail: jkochman@wum.edu.pl

Received: 17.05.2021, accepted: 1.07.2021. 


\section{Classifications, phenotypes and sex differences}

The most prevalent BAV phenotype, found in $70 \%$ of patients, results from fusion of the left and right coronary cusp (LCC and RCC). 10-20\% of patients present with fusion of the RCC and the non-coronary cusp (NCC) and $5-10 \%$ have fusion of the LCC with NCC. The most common anatomical BAV classification was presented by Sievers and Schmidtke based on 304 surgical specimens (Figure 1) [11]. Presence of two cusps and two commissures with no fusion raphe is considered type 0 . In type 1 BAV raphe fusing two cups are present, whereas two raphes can be found in type 2 BAV.

Recently, a new classification, based on multidetector computed tomography (CT), has been proposed, where the authors focused on the number of commissures and the presence of raphe, distinguishing 3 BAV morphologies [12]. In tricommissural (functional or acquired) morphology, there is a complete fusion of one commissure between two cusps, while 3 clearly distinguishable commissures can still be identified. Bicommissural raphe type presents with fibrous or calcified raphe fusing 2 cusps at their basal part. In contrast, in the bicommissural non-raphe type there is a complete fusion of 2 cusps without any visible ridge. The authors describe several additional anatomical differences important for the TAVI procedure between these phenotypes. For instance, when compared to tricommissural BAV, bicommissural valves had larger intercommissural distances, as well as dimensions of the ascending aorta, sinotubular junctions and amount of calcifications. These different morphological presentations must be taken into account when planning and performing a TAVI procedure. Stenotic BAV without raphe in a 70-year-old man may require a different approach than stenotic BAV with severely calcified raphe in an 85-year-old woman, and procedure planning, choice of the bioprosthesis, and the final result can differ profoundly between the patients.

The impact of different phenotypes on TAVI outcomes has recently been demonstrated in a prospective registry of 1034 patients with BAV stenosis, where a combina- tion of calcified raphe and severe leaflet calcifications was identified as a high-risk phenotype [13]. The 2-year all-cause mortality in this subset of patients was significantly higher compared to those who had only one or did not have any of these features (25.7\% vs. 9.5\% vs. 5.9\%; respectively; log-rank $p<0.001)$. This high risk phenotype, present in $25 \%$ of the study population, was also associated with a 3-fold higher rate of aortic root injury $(p<0.001)$, moderate-to-severe paravalvular regurgitation $(p=0.002)$, and higher 30-day mortality $(p=0.016)$. These differences were also observed when surgical classification was applied. The 2-year all-cause mortality was much higher in patients with type 1 and type 2 morphology as compared to those with type 0 (13.5\% vs. $2.4 \%, p=0.04)$. Observations from the above mentioned studies clearly indicate that careful preprocedural planning including the analysis of the valve phenotype in the CT scan can improve patient selection and may have a major impact on TAVI outcome. It could also imply wider utilization of cerebral protection devices, as presence of severe calcifications has been identified as a risk factor for periprocedural stroke [14]. This leads us to the conclusion that not only 'simple' differentiation between TAV and BAV in general, but also the distinction between different BAV morphologies, needs to be taken into account when TAVI is to be performed.

The CT images and reconstructions of different BAV morphologies are presented in Table I [15].

\section{TAVI outcomes in patients with BAV stenosis}

Traditionally, patients with BAV have been excluded from randomized clinical trials comparing TAVI to surgical aortic valve replacement (SAVR), as BAV was considered a relative contraindication for TAVI $[16,17]$. The main concerns comprised higher risk of bioprosthesis under-expansion, significant paravalvular leak (PVL) due to severe calcification, increased risk for aortic root rupture, coronary occlusion and quicker leaflet degeneration [13]. An example of these problems with BAV is presented in Figure 2. However, new generation TAVI devices seem to
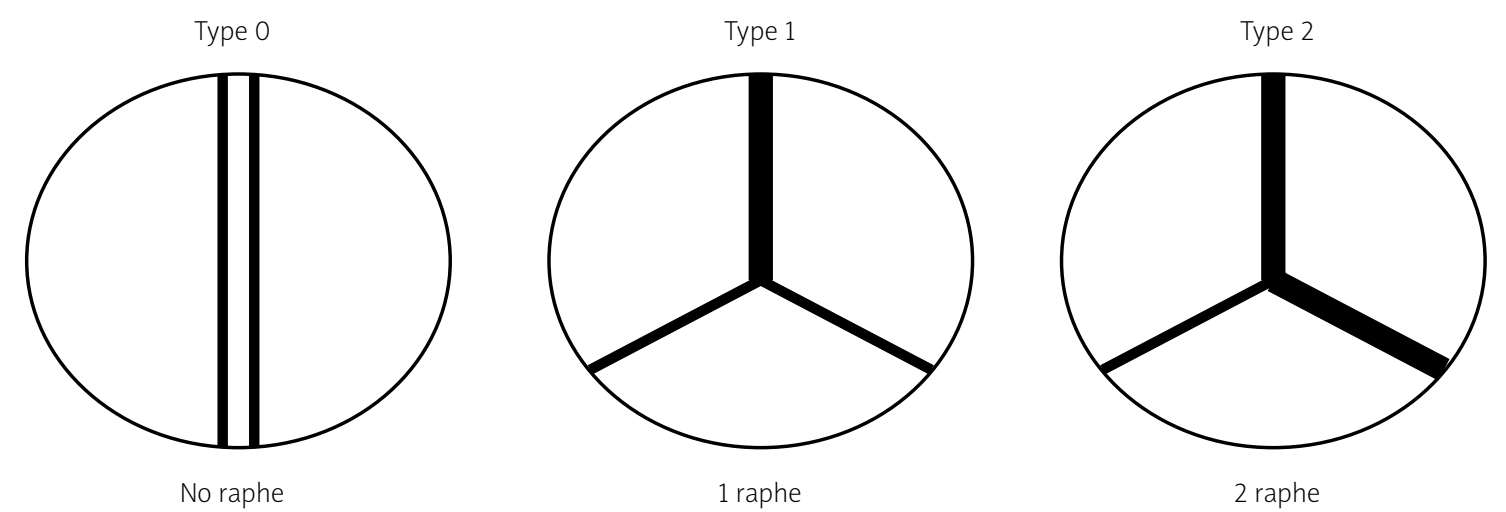

Figure 1. Bicuspid valve classification. Figure adapted from Sievers and Schmidtke [11] 
Table I. Computed tomography (CT) images and reconstructions of different bicuspid aortic valve (BAV) morphologies. Table modified according to the Consensus Document of the Society of Cardiovascular Computed Tomography [15]

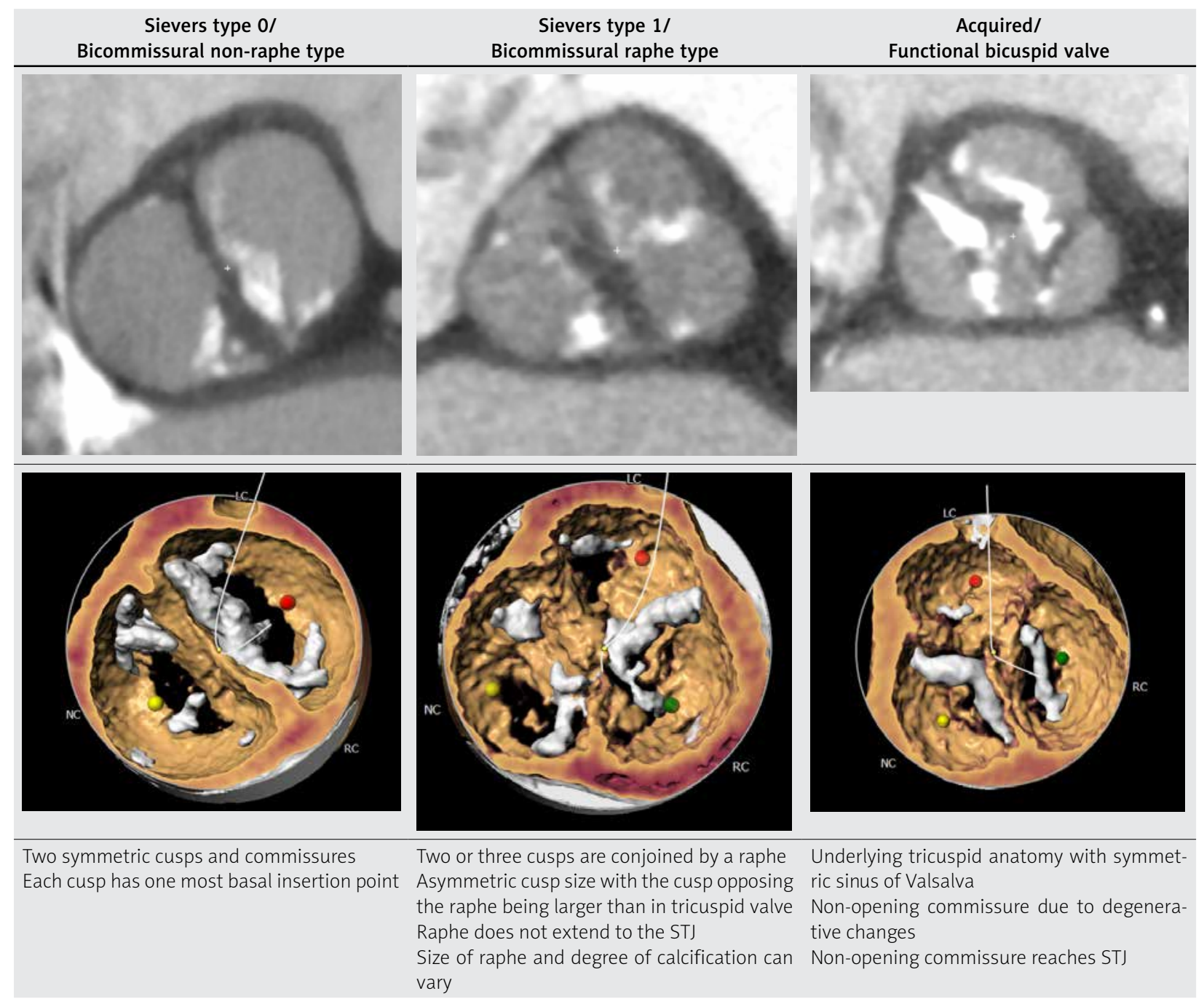

STJ - sinotubular junction.

offer similar clinical outcomes (mortality and stroke) in both BAV and TAV patients at 1- to 2-year follow-up, partly due to better apposition with repositionable and/or sealing features $[18,19]$. PVL and permanent pacemaker implantation (PPI) rates in BAV are also significantly lower than with first generation TAVI devices [20].

An interesting insight in the topic of the new generation devices comes from the recently published study of 170959 patients undergoing TAVI, where BAV was present in 5412 patients and 3705 procedures were performed with the use of current-generation bioprosthesis [21]. Compared to the previous generation, device success increased (93.5\% vs. $96.3 \% ; p=0.001)$ and the incidence of moderate-to-severe aortic insufficiency was significantly lower $(14.0 \%$ vs. $2.7 \% ; p<0.001)$ when new-generation valves were used. Device success with current-generation prostheses was only marginally lower in the BAV group versus the TAV group $(96.3 \%$ in bicus- pid versus $97.4 \%$ in tricuspid, $p=0.07$ ), with a higher incidence of more than moderate aortic regurgitation in BAV patients $(2.7 \%$ vs. $2.1 \% ; p<0.001)$. The BAV cohort had slightly lower risk of adjusted 1-year mortality (hazard ratio $(\mathrm{HR})=0.88,95 \%$ confidence interval $(95 \% \mathrm{Cl})$ : $0.78-0.99)$ with no difference in the 1-year adjusted risk of stroke ( $\mathrm{HR}=1.1495 \% \mathrm{Cl}: 0.94-1.39)$. Another study based on the STS Registry evaluated the efficacy of Evolut PRO and Evolut $R$ valves by comparing outcomes in 929 pairs of matched patients with BAV and TAV [9]. There were no significant differences in all-cause mortality at 30 days $(2.6 \%$ vs. $1.7 \% ; p=0.18)$ and 1 year $(10.4 \%$ vs. $12.1 \% ; p=0.63)$ between the groups. The incidence of stroke at 30 days (3.4\% vs. $2.7 \% ; p=0.41)$ and at 1 year (3.9\% vs. $4.4 \% ; p=0.93)$ was also comparable.

Makkar et al. analyzed 2691 propensity-score matched pairs of patients with bicuspid and tricuspid aortic stenosis undergoing TAVI [7]. There was no signif- 

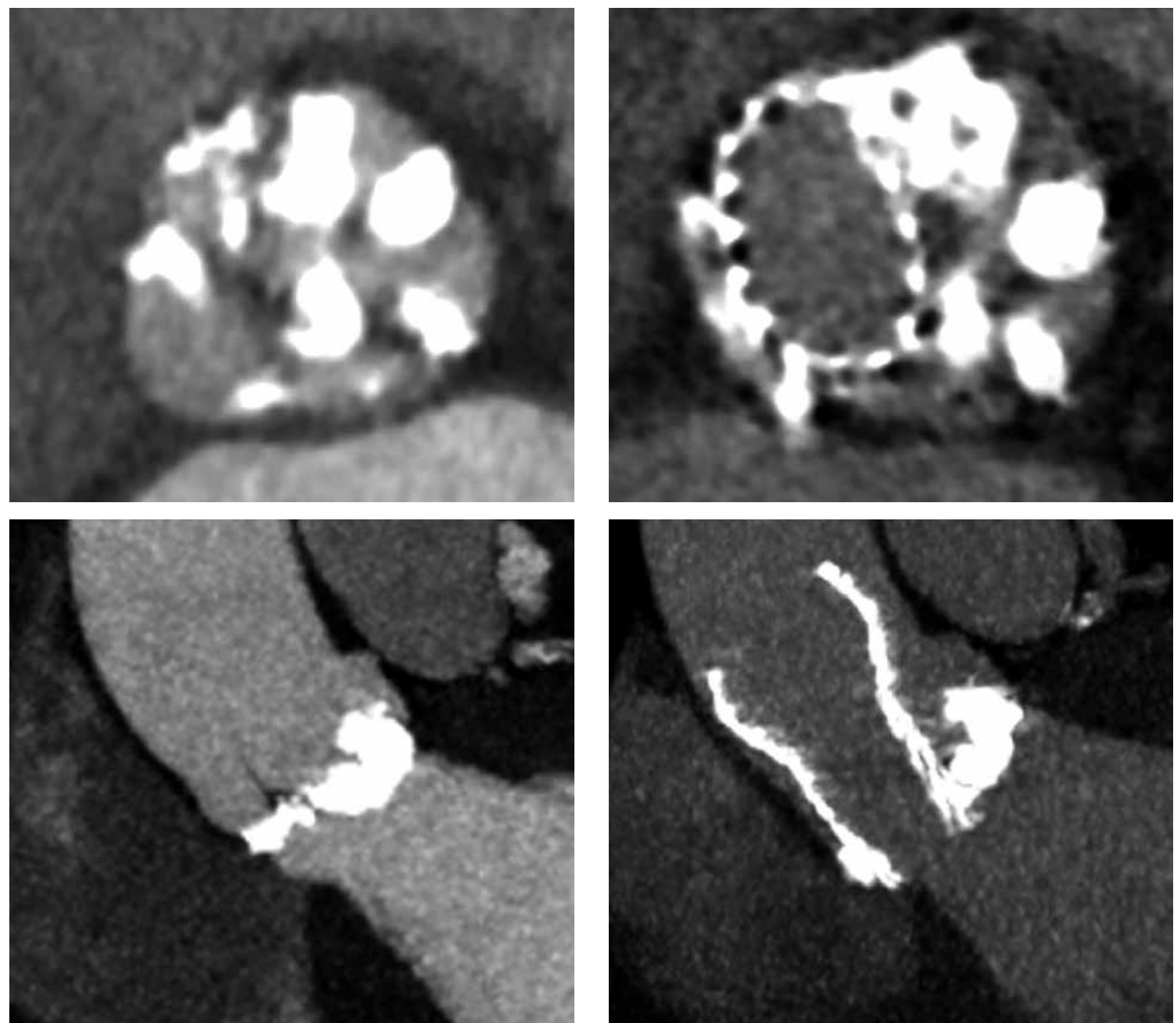

Figure 2. Pre- and post- transcatheter aortic valve implantation (TAVI) computed tomography images that present severe calcifications in bicuspid aortic valve and problems with proper expansion and elliptical shape of the prosthesis

icant difference in all-cause mortality at 30 days $(2.6 \%$ vs. $2.5 \%$; $\mathrm{HR}=1.04,95 \% \mathrm{Cl}: 0.74-1.47)$ or 1 year $(10.5 \%$ vs. $12.0 \%$; $\mathrm{HR}=0.90,95 \% \mathrm{Cl}: 0.73-1.10)$. However, the 30 -day stroke risk was higher in BAV patients $(2.5 \%$ vs. $1.6 \% ; \mathrm{HR}=1.57,95 \% \mathrm{Cl}: 1.06-2.33)$, as was the rate of possibly fatal periprocedural complications requiring cardiac surgery ( $0.9 \%$ vs. $0.4 \%$; absolute risk difference (RD) $0.5 \%, 95 \% \mathrm{Cl}: 0-0.9 \%)$. This was mostly due to 7 cases of annulus rupture in the BAV group compared to 0 cases in the tricuspid cohort. There was no significant difference in the PVL rate at 30 days (2.0\% of patients with BAV and $2.4 \%$ with TAV; absolute RD $=0.3 \%, 95 \% \mathrm{Cl}:-1.3 \%$ to $0.7 \%)$ or 1 year. The authors observed an increased risk of PPI at 30 days in the bicuspid cohort (9.1\% vs. $7.5 \%$; absolute RD $=1.65 \%, 95 \% \mathrm{Cl}: 1.63-1.66 \% ; \mathrm{HR}=1.23$, $95 \% \mathrm{Cl}: 1.02-1.49)$. Valve hemodynamics were similar in both groups.
Similar results were observed in a metanalysis of 13 studies comparing TAVI outcomes in TAV and BAV patients [22]. 30-day and 1-year mortality did not differ between the groups (30-day OR $=1.13 ; 95 \% \mathrm{Cl}$ : $0.88-$ $1.46, p=0.33)(1$-year $\mathrm{OR}=1.02 ; 95 \% \mathrm{Cl}: 0.77-1.37$, $p=0.87)$. Also, the stroke prevalence was similar in both cohorts. However, BAV was associated with higher risk of conversion to surgery $(\mathrm{OR}=2.35 ; 95 \% \mathrm{Cl}: 1.30-4.23$, $p=0.005)$, more than mild PVL $(\mathrm{OR}=1.67 ; 95 \% \mathrm{Cl}$ : $1.29-2.17 ; p=0.0001$ ), second valve implantation (OR $=2.06 ; 95 \% \mathrm{Cl}: 1.31-3.25 ; p=0.002)$ and failure of the device $(\mathrm{OR}=1.26 ; 95 \% \mathrm{Cl}: 1.02-1.56 ; p=0.04)$. Notably, the number of complications declined in patients treated with new generation devices, but discrepancies in the outcomes persisted. Percentages of the most important TAVI complications with the use of modern generation prostheses in the bicuspid valve cohort 
based on the metanalysis by Ueshima et al. are shown in Figure 3 [22].

\section{Prosthesis types and TAVI outcomes}

Comparison of TAVI outcomes between self-expanding (SE) and balloon-expandable (BE) valves in patients with $\mathrm{BAV}$, based on the data from the above mentioned metanalysis, is shown in Figure 4. There was no differences in 30-day or 1-year mortality although BE prostheses were associated with a lower risk of second valve and pacemaker implantation, but also with a higher risk of annular rupture [23]. One may speculate that the generally observed increased rate of PPI in BAV patients is associated with asymmetric leaflet calcifications, calcified raphe and aortomitral continuity, which impairs the appropriate height of implantation. In these cases, greater forces are applied to the basal part of the septum and conduction system, which results in conduction disturbances. Moreover, aggressive oversizing, especially in self-expanding valves, may further exaggerate this problem. However, in the study of Perlman et al. greater oversizing (>10\%) of Sapien 3 BE prostheses was not associated with a higher PPI rate [24]. Deep prosthesis position (OR $=5.55 ; 95 \% \mathrm{Cl}: 1.18-26.19 ; p=0.03)$ and coexistence of coronary cusp fusion and a calcium bridge $(\mathrm{OR}=7.69 ; 95 \% \mathrm{Cl}: 2.08-28.46 ; p=0.002)$ have been identified as independent predictors for PPI requirement.

The combination of asymmetric leaflet calcifications and a calcified raphe could also result in bioprosthesis underexpansion and significant PVL. An anatomical study of patients after elective aortic valve replacement revealed that proper circular deployment of the prosthesis stents was much less frequent in BAV than TAV, with $79 \%$ of the prostheses having non-circular shape [25]. Such adverse features of bicuspid valves often result in the need for post-dilatation, which can help in adequate prosthesis implantation and reduction of aortic regurgitation. The need for post-dilatation increases with the

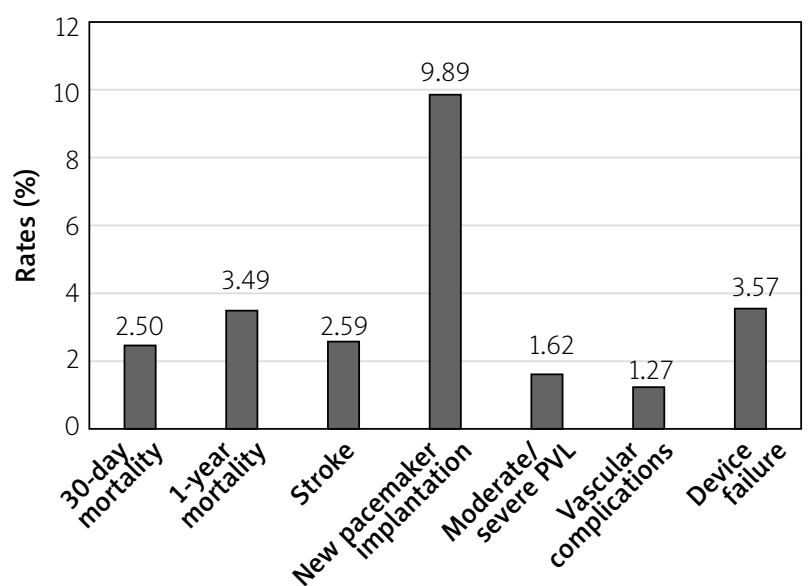

Figure 3. Percentage of most important transcatheter aortic valve implantation complications with the use of modern generation prostheses in bicuspid valve cohort based on Ueshima metanalysis [22]

amount of calcifications. This is also one of the factors that increases the complexity and length of the TAVI procedure in BAV patients.

In the BEAT (Balloon versus self-expandable valve for the treatment of bicuspid aortic valve stenosis) registry, 353 consecutive patients with BAV were treated with TAVI using contemporary Evolut R/PRO (SE) or Sapien 3 (BE) prostheses [26]. Two hundred and forty-two (68.6\%) patients received the Sapien 3 and 111 (68.6\%) the Evolut R or PRO. Device success according to Valve Academic Research Consortium-2 criteria was achieved in $85.6 \%$ of Sapien 3 and $87.2 \%$ of Evolut R/PRO cases ( $p=0.68$ ). After propensity-score matching, the PVL rate at 1 was higher in SE valves as compared to BE valves (9.3\% vs. $0.0 \%, p=0.043)$. No significant difference was found in the number of pacemakers implanted in both cohorts (SE $16.0 \%$ vs. BE $16.1 \% ; p=0.977$ ). SE valves tended to have better hemodynamics at discharge and after 1 year (lower mean gradient and larger effective orifice area). There were 4 cases of annular rupture in the BE cohort

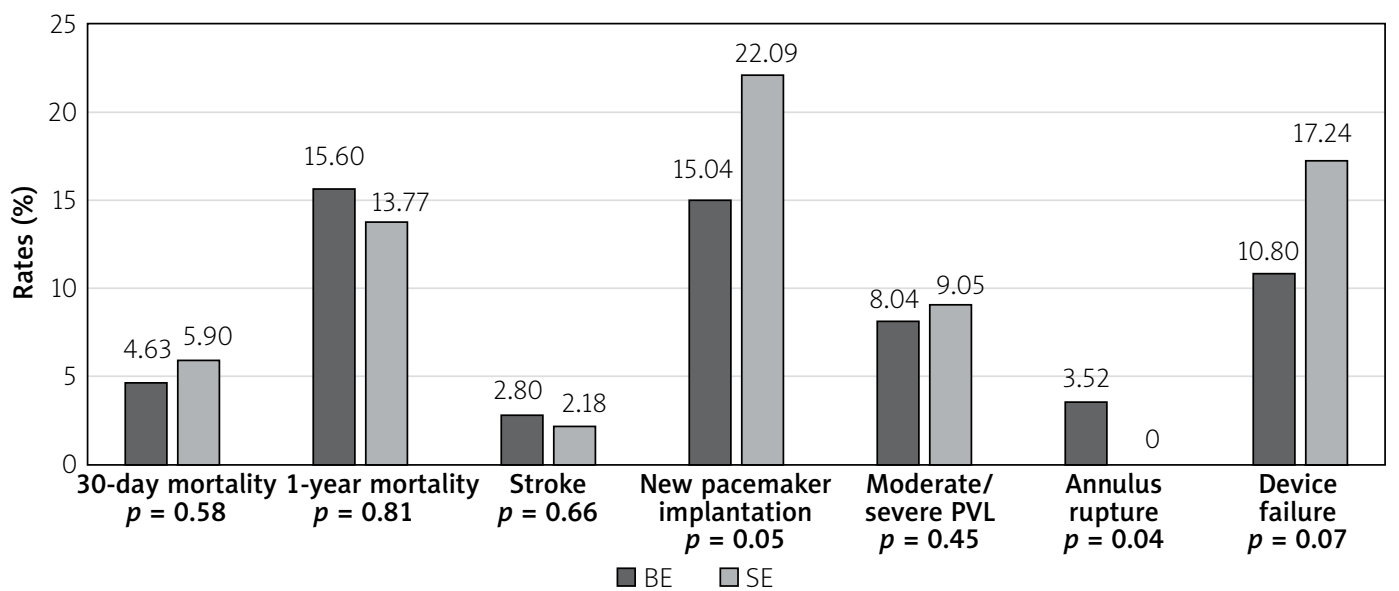

Figure 4. Comparison of transcatheter aortic valve implantation outcomes between self-expanding and balloon-expandable valves in patients with bicuspid aortic valve. Based on data from Ueshima metanalysis [22] 
and 0 in the SE cohort, but this difference did not reach statistical significance. Clinical events and mortality rates after 30 days and 1 year in both groups were similar, also in the propensity score matched population. The need for post-dilatation was significantly higher in SE valves than $\mathrm{BE}$ valves ( $45.9 \%$ vs. $14.5 \%$; $p=0.001$ ), which may be a result of underexpansion of the valve directly after implantation. It seems that SE valves usually obtain an irregular BAV orifice but are less capable of achieving a proper circular shape. In a recently published study where self-expanding Evolut $R$ and Evolut Pro valves were utilized, no differences between BAV and TAV patients in terms of total mortality, stroke, or valve hemodynamics were observed, although the procedure time was longer and the reoperation rate was higher in BAV patients [9]. Following this study, Medtronic received a CE mark for its valves in intermediate, high, and extremely high-risk BAV patients.

\section{Sizing}

The matter of choosing an adequate valve size in BAV stenosis is of utmost importance, and a few measurement methods have been proposed. The most commonly applied one is based on conventional annular sizing, whereas others take into account the inter-commissural distance and supra-annular sizing. In the BAVARD retrospective registry, Tchetche et al. retrospectively analyzed pre- and post-TAVI CT of 101 BAV and 88 TAV patients. Type 0 was identified in $12.9 \%$ and type 1 in $86.1 \%$ of the study population. The objective of the study was to capture the sizing ratios used for TAVI in BAV, evaluate the prostheses' geometry after implantation and compare the results with the TAV cohort [23]. The authors found no significant changes in PVL and PPM between BAV and TAV groups. Diameters of the implanted valves from the distal edge to $12 \mathrm{~mm}$ above the edge and the reshaping of the aortic annulus were consistent in TAV and BAV. The authors observed a tendency to prosthesis underexpansion in BAV. They concluded that sizing at the annulus level with minimal oversizing was a reliable method in most of the type 0 and type 1 bicuspid valves. Supra-annular sizing could be used in cases when the mean perimeter-derived diameter of the annulus is larger than the intercommissural distance, which is called tapered configuration.

Another study was designed to compare the results of sizing based on annular area (AA) versus supra-annular area (SA) in BAV [27]. The results showed significant variability between the valve size suggested by both methods. AA sizing proved to be more adequate for the final size of the valve implanted. In patients in whom the SA method suggested that another size should be used, no increase in clinical and valve related events was observed. In conclusion, the authors suggested that the SA method in BAV should no longer be pursued. Petronio et al. proposed another method called CASPER: Calcium Algorithm Sizing for bicusPid Evaluation with Raphe [28]. This approach is focused on 3 features: 1) calcium score, 2) raphe length with respect to annulus diameter, 3) calcium localization in relation to raphe. At the first step the annulus diameter is measured. A calcium score above 300 results in detraction of $1 \mathrm{~mm}$ from the derived diameter. A raphe longer than $50 \%$ of the annulus diameter requires subtraction of another $1 \mathrm{~mm}$. With the higher burden of calcium on the raphe site, one should subtract another $0.5 \mathrm{~mm}$ to get a proper size of the prosthesis. The method was validated on 21 consecutive patients and good results were obtained in all procedures. Figure 5 shows basic differences in TAV and BAV sizing.

\section{Conclusions}

The general conclusion from the contemporary studies, although no long-term observations after TAVI in BAV are available yet, is that short- and mid-term outcomes with the use of new generation prostheses are similar in patients with bicuspid and tricuspid valves. Nevertheless, the decision on how to treat a patient with symptomatic BAV stenosis should always be based on a very
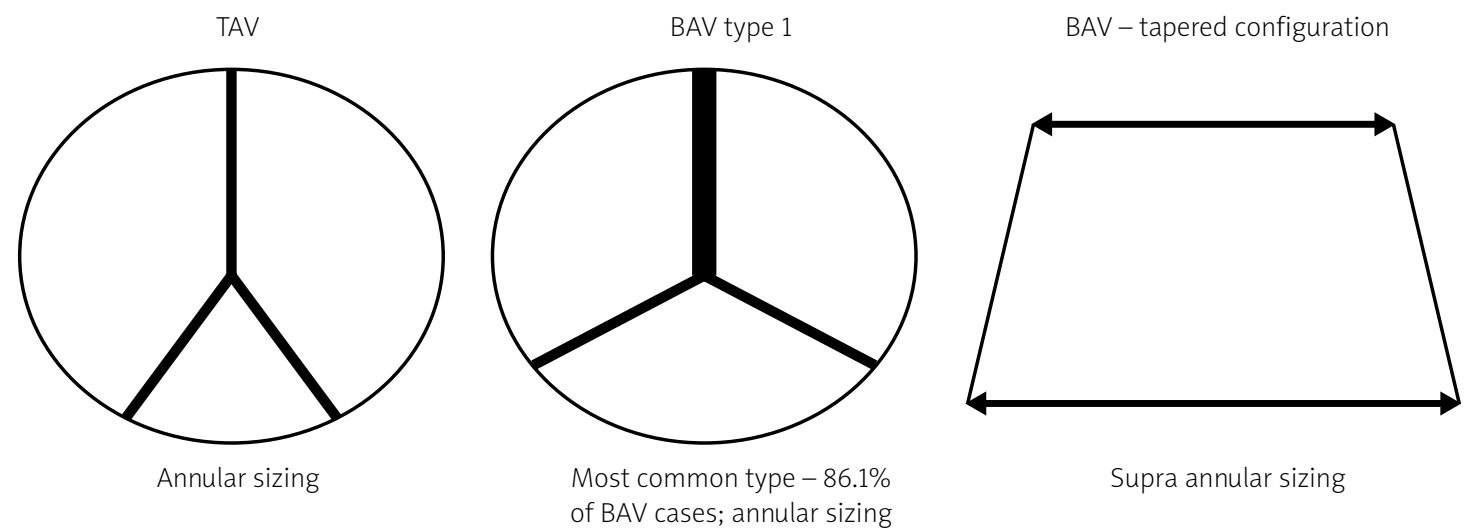

Figure 5. Sizing differences in bicuspid aortic valve (BAV) and tricuspid aortic valve (TAV). Adapted from Tchetche et al. [23] 
careful analysis of clinical data, anatomical characteristics obtained from pre-procedural imaging and, last but not least, the operator's experience. It needs to be underlined that there are still many unresolved questions, as most of the trials do not evaluate data of BAV morphologies, which could be crucial to facilitate the proper patient selection. In particular, type 0 in the Sievers classification, which is considered "true" BAV and is usually present in younger patients, was not equally represented in registries. The optimal sizing method is also of concern, although it seems that valve implantation based on the annular sizing results in selection of larger valves, yielding larger effective orifice areas and a lower gradient. The safety of TAVI in younger, low-risk patients with BAV is questionable, as pacemaker implantation rates after the procedure remain high. Some of these unresolved issues could possibly be addressed in a randomized trial comparing SAVR vS TAVI in this population. The need to perform such a study becomes even more important considering the anticipated rapid growth of TAVI procedures in BAV patients that is likely to follow the upcoming expansions of indications for TAVI to a younger and healthier population. Unfortunately, the chances that it will be performed in the forthcoming years are rather low, taking into account its difficult design and possible financing problems.

\section{Conflict of interest}

The authors declare no conflict of interest.

\section{References}

1. Borger MA, Fedak PWM, Stephens EH, et al. The American Association for Thoracic Surgery consensus guidelines on bicuspid aortic valve-related aortopathy: Full online-only version. J Thoracic Cardiovasc Surg 2018; 156: e41-74.

2. Beppu S, Suzuki S, Matsuda H, et al. Rapidity of progression of aortic stenosis in patients with congenital bicuspid aortic valves. Am J Cardiol 1993; 71: 322-7.

3. Masri A, Kalahasti V, Alkharabsheh S, et al. Characteristics and long-term outcomes of contemporary patients with bicuspid aortic valves. J Thoracic Cardiovasc Surg 2016; 151: 1650-9.e1.

4. Liu X, He Y, Zhu Q, et al. Supra-annular structure assessment for self-expanding transcatheter heart valve size selection in patients with bicuspid aortic valve. Catheter Cardiovasc Interv 2018; 91: 986-94.

5. Tzemos N, Therrien J, Yip J, et al. Outcomes in adults with bicuspid aortic valves. JAMA 2008; 300: 1317-25.

6. Michelena HI, Desjardins VA, Avierinos JF, et al. Natural history of asymptomatic patients with normally functioning or minimally dysfunctional bicuspid aortic valve in the community. Circulation 2008; 117: 2776-84.

7. Makkar RR, Yoon SH, Leon MB, et al. Association between transcatheter aortic valve replacement for bicuspid vs tricuspid aortic stenosis and mortality or stroke. JAMA 2019; 321: 2193-202.

8. Attinger-Toller A, Bhindi R, Perlman GY, et al. Mid-term outcome in patients with bicuspid aortic valve stenosis following transcatheter aortic valve replacement with a current generation device: a multicenter study. Catheter Cardiovasc Interv 2020; 95: 1186-92.

9. Forrest JK, Kaple RK, Ramlawi B, et al. Transcatheter aortic valve replacement in bicuspid versus tricuspid aortic valves from the STS/ACC TVT registry. JACC Cardiovasc Interv 2020; 13: 1749-59.

10. Pineda AM, Rymer J, Wang A, et al. Transcatheter aortic valve replacement for patients with severe bicuspid aortic stenosis. Am Heart J 2020; 224: 105-12.

11. Sievers HH, Schmidtke C. A classification system for the bicuspid aortic valve from 304 surgical specimens. J Thoracic Cardiovasc Surg 2007; 133: 1226-33.

12. Jilaihawi $H$, Chen $M$, Webb J, et al. A bicuspid aortic valve imaging classification for the TAVR era. JACC Cardiovasc Imaging 2016; 9: 1145-58.

13. Philip F, Faza NN, Schoenhagen P, et al. Aortic annulus and root characteristics in severe aortic stenosis due to bicuspid aortic valve and tricuspid aortic valves: implications for transcatheter aortic valve therapies. Catheter Cardiovasc Interv 2015; 86: E88-98.

14. Ndunda PM, Vindhyal MR, Muutu TM, Fanari Z. Clinical outcomes of sentinel cerebral protection system use during transcatheter aortic valve replacement: a systematic review and meta-analysis. Cardiovasc Revasc Med 2020; 21: 717-22.

15. Blanke P, Weir-McCall JR, Achenbach S, et al. Computed tomography imaging in the context of transcatheter aortic valve implantation (TAVI)/transcatheter aortic valve replacement (TAVR): an Expert Consensus Document of the Society of Cardiovascular Computed Tomography. JACC Cardiovasc Imaging 2019; 12: 1-24.

16. Rodríguez-Caulo EA, Araji OA, Barquero JM. Transapical aortic valve implantation in bicuspid aortic valves: must be an absolute contraindication? Res Cardiovasc Med 2012; 1: 37-9.

17. Mack MJ, Leon MB, Thourani VH, et al. Transcatheter aortic-valve replacement with a balloon-expandable valve in low-risk patients. N Engl J Med 2019; 380: 1695-705.

18. Yoon SH, Bleiziffer S, De Backer O, et al. Outcomes in transcatheter aortic valve replacement for bicuspid versus tricuspid aortic valve stenosis. J Am Coll Cardiol 2017; 69: 2579-89.

19. Tchetche D, Van Mieghem NM. New-generation TAVI devices: description and specifications. Eurointervention 2014; 10 Suppl U: U90-100.

20. Yoon SH, Lefèvre T, Ahn JM, et al. Transcatheter aortic valve replacement with early- and new-generation devices in bicuspid aortic valve stenosis. J Am Coll Cardiol 2016; 68: 1195-205.

21. Halim SA, Edwards FH, Dai D, et al. Outcomes of transcatheter aortic valve replacement in patients with bicuspid aortic valve disease: a report from the Society of Thoracic Surgeons/American College of Cardiology Transcatheter Valve Therapy Registry. Circulation 2020; 141: 1071-9.

22. Ueshima D, Nai Fovino L, Brener SJ, et al. Transcatheter aortic valve replacement for bicuspid aortic valve stenosis with firstand new-generation bioprostheses: a systematic review and meta-analysis. Int J Cardiol 2020; 298: 76-82.

23. Tchetche D, de Biase C, van Gils L, et al. Bicuspid aortic valve anatomy and relationship with devices: the BAVARD Multicenter Registry. Circ Cardiovasc Interv 2019; 12: e007107.

24. Perlman GY, Blanke P, Dvir D, et al. Bicuspid aortic valve stenosis: favorable early outcomes with a next-generation transcatheter heart valve in a multicenter study. JACC Cardiovasc Interv 2016; 9: 817-24. 
25. Zegdi R, Ciobotaru V, Noghin M, et al. Is it reasonable to treat all calcified stenotic aortic valves with a valved stent? Results from a human anatomic study in adults. J Am Coll Cardiol 2008; 51: 579-84.

26. Mangieri A, Tchetche D, Kim WK, et al. Balloon versus self-expandable valve for the treatment of bicuspid aortic valve stenosis: insights from the BEAT International Collaborative Registrys. Circ Cardiovasc Interv 2020; 13: e008714.

27. Weir-McCall JR, Attinger-Toller A, Blanke P, et al. Annular versus supra-annular sizing for transcatheter aortic valve replacement in bicuspid aortic valve disease. J Cardiovasc Comput Tomogr 2020; 14: 407-13.

28. Petronio AS, Angelillis M, De Backer O, et al. Bicuspid aortic valve sizing for transcatheter aortic valve implantation: development and validation of an algorithm based on multi-slice computed tomography. J Cardiovasc Comput Tomogr 2020; 14: 452-61. 